\title{
Novelty vs. familiarity principles in preference decisions: task-context of past experience matters
}

\author{
Hsin-I Liao ${ }^{1,2,3}$, Su-Ling Yeh ${ }^{1,4}$ and Shinsuke Shimojo ${ }^{2,3,5 *}$ \\ Department of Psychology, National Taiwan University, Taipei, Taiwan \\ 2 Division of Biology, California Institute of Technology, Pasadena, CA, USA \\ 3 Shimojo Implicit Brain Function Project, Exploratory Research for Advanced Technology, Japan Science and Technology Agency, Kawaguchi, Japan \\ ${ }^{4}$ Center for Neurobiology and Cognitive Science, National Taiwan University, Taipei, Taiwan \\ ${ }^{5}$ Computation and Neural Systems, Division of Biology, California Institute of Technology, Pasadena, CA, USA
}

\section{Edited by:}

Emmanuel Pothos, Swansea

University, UK

Reviewed by:

Richard J. Tunney, University of Nottingham, UK

Christoph T. Weidemann, Swansea

University, UK

\section{${ }^{*}$ Correspondence:}

Shinsuke Shimojo, Division of Biology, California Institute of Technology, 1200 East California Boulevard, Pasadena, CA 91125, USA.

e-mail:sshimojo@caltech.edu
Our preferences are shaped by past experience in many ways, but a systematic understanding of the factors is yet to be achieved. For example, studies of the mere exposure effect show that experience with an item leads to increased liking (familiarity preference), but the exact opposite tendency is found in other studies utilizing dishabituation (novelty preference). Recently, it has been found that image category affects whether familiarity or novelty preference emerges from repeated stimulus exposure (Park et al., 2010). Faces elicited familiarity preference, but natural scenes elicited novelty preference. In their task, preference judgments were made throughout all exposures, raising the question of whether the task-context during exposure was involved. We adapt their paradigm, testing if passive exposure or objective judgment task-contexts lead to different results. Results showed that after passive viewing, familiar faces were preferred, but no preference bias in either direction was found with natural scenes, or with geometric figures (control). After exposure during the objective judgment task, familiar faces were preferred, novel natural scenes were preferred, and no preference bias was found with geometric figures. The overall results replicate the segregation of preference biases across object categories and suggest that the preference for familiar faces and novel natural scenes are modulated by taskcontext memory at different processing levels or selection involvement. Possible underlying mechanisms of the two types of preferences are discussed.

Keywords: preference, memory, social cognition, judgment, perception

\section{INTRODUCTION}

Experience affects our preferences in a variety of different, sometimes contradictory ways. Our preferences sometimes lean toward things we have not previously experienced, such as novel new products or shops. On the other hand, we sometime prefer things because we have extensive experience with them, such as familiar faces or items that evoke nostalgia (e.g., Houston-Price and Nakai, 2004). Scientific research has generally uncovered and investigated these trends separately. In the mere exposure effect (Zajonc, 1968, 2001), familiar things are preferred over novel ones. Meanwhile other studies utilize effects such as dishabituation, where novel visual objects/places are preferred (e.g., Fantz, 1964; Berlyne, 1970; Olst, 1971; Bevins and Bardo, 1999; Klebaur and Bardo, 1999).

The results may have varied because they had been obtained from different paradigms, subjects, and species. In a recent study that directly opposed the two effects within a single paradigm, Park et al. (2010) showed that the two bases of preference, novelty and familiarity, have different patterns of dominance across different object categories. In a visual comparison paradigm, participants rated their relative preference between a pair of pictures presented side-by-side. In a given experimental sub-block, one of the pictures was presented in every trial, and thus became increasingly familiar. The other picture was new for each trial, and so was always novel. Three different picture types were tested: faces, natural scenes, and geometric figures, with each image category tested separately. For face stimuli, repeated faces became increasingly preferred as the sub-block progressed. Meanwhile, for natural scenes, new stimuli quickly became preferred over the old one. For geometric figures, no strong preference bias was found in either direction.

While this segregation of novelty vs. familiarity preference between faces and natural scenes was replicated across a wide range of conditions in Park et al. (2010), the preference for novel natural scenes is inconsistent with studies of the mere exposure effect (e.g., Zajonc, 1968; Zajonc et al., 1972; Bornstein, 1989). Based on these studies, the preference for the repeated stimulus should have increased rather than decreased. This inconsistency cannot be explained based on stimulus types, since studies have shown the mere exposure effect to exist across among a wide range of stimulus types, such as words (Zajonc, 1968; Monahan et al., 2000; Topolinski and Strack, 2009; Garcia-Marques et al., 2010), photographs (Bornstein and D'Agostino, 1992; Crisp et al., 2009), polygons (Bornstein and D'Agostino, 1992), random-dot patterns (de Vries et al., 2010), line drawings (Hupbach et al., 2006), and most relevantly, colorful paintings (Zajonc et al., 1972) which are similar to the natural scene pictures used in Park et al. (2010).

There is one noticeable difference in procedure that may account for the difference, which is the differing task-contexts in which the repeated pictures were experienced. In Park et al. (2010), participants were requested to make preference judgments with each stimulus presentation. Meanwhile, mere exposure experiments 
typically present the repeated stimulus in a passive viewing context (hence "mere" exposure), with preference judgments being elicited only after the exposure period (e.g., Zajonc et al., 1972; Bornstein and D'Agostino, 1992; Monahan et al., 2000). Performing a preference judgment on each presentation may alter the depth and/or type of processing of the stimulus and thus may change the effects of exposure on preference.

For instance, it is possible that the explicit evaluation task performed in each trial leads to stronger habituation over repeated stimuli, offsetting the mere exposure effect and leading to a novelty preference for natural scenes. Such an effect of processing depth (Schneider and Shiffrin, 1977; Shiffrin and Schneider, 1977) is hypothesized to have influence on preferences that familiarity preference depends on automatic processing, whereas novelty preference requires more controlled cognitive processing. Accordingly, tendencies toward familiarity preference would require only passive exposures, whereas tendencies toward novelty preference would require active judgment during exposure. This hypothesis would predict that changing Park et al.'s (2010) procedure to passive viewing of the stimuli should reduce or abolish the novelty preference for natural scenes.

Another hypothesis is that what is critical is not so much of depth of processing but rather type of processing: that is, different types of tasks may elicit different types of memory processing, leading to different effects on novelty and familiarity preferences. It may be the active evaluation of preference in particular that leads to the novelty preference. Such an effect of processing type is suggested by a combination of studies. Shimojo et al. (2003) showed that patterns of gaze orienting contribute to preference judgment, but not as much to other, objective types of judgment. They hypothesized that there exists a feedback loop between gaze orienting and preference judgments in particular. Because gaze orientation is attracted by novel visual stimuli (Fantz, 1964; Wu and Remington, 2003), the active engagement of gaze mechanisms by an explicit preference choice task may enhance a preference for novel stimuli. This alternative hypothesis would predict that changing Park et al.'s (2010) procedure to use an objective judgment during the exposure phase would reduce the novelty preference, as objective judgment engages the gaze mechanisms to a lesser extent.

Therefore the current study examines whether the familiarity and the novelty preferences found in Park et al. (2010) are based on different mechanisms, and how the level and/or type of processing during exposure matters in that regard. The results will hopefully assist us in resolving the inconsistency of the results in the literature. In two experiments, we manipulated task presence and task type during exposure to examine whether and how the hypothesized factors affect familiarity and novelty preferences in a subsequent preference test. The experiments use the same stimuli and methods as Park et al. (2010), but we divide the experiment into two phases: an experience phase followed by a preference judgment phase. In Experiment 1, passive viewing of stimuli with no preference judgment was conducted during the experience phase, as in the typical mere exposure effect study, to see if preferences are modulated by depth of processing. In Experiment 2, we restored the presence of an explicit judgment task, but instead of a preference judgment, we used an objective judgment task (comparing either stimuli roundness, color, or complexity) to see if preferences are modulated by type of processing. We examine whether introducing these different task-contexts of stimulus experience modulates the preference for familiar faces and novel natural scenes found in Park et al. (2010).

\section{EXPERIMENT 1: PRESENCE OR ABSENCE OF A JUDGMENT TASK}

This experiment aims to examine whether the segregation of preference for familiar faces and novel natural scenes can be formed under conditions of mere exposure. In the experience phase, participants were not requested to do any judgment but just to watch the pairs of stimuli. Afterward, in a preference judgment phase, participants were given an unexpected task to rate their relative preference between the two items in each subsequent trial. We measured whether there was any preference bias toward familiar faces or novel natural scenes immediately after the exposure.

Both the mere exposure effect and the original results from Park et al. (2010) indicate that we should expect the familiar faces to be preferred over novel ones. For natural scenes, the expected results of mere exposure effect and Park et al. (2010) diverge. If Park et al.'s (2010) findings of novelty preference were caused by the presence of a judgment task during the exposure phase, then we would expect the present manipulation to cause a reversion to the familiarity preference found in the mere exposure effect.

\section{METHODS \\ Participants}

Twenty adults (graduates, undergraduates, and staff at Caltech or National Taiwan University) who were naïve about the purpose of this study participated in this experiment. Three were Caucasians, one was Latino, and 16 were Asians. The Caltech or NTU Committee for the Protection of Human Subjects approved the experiment and the informed consent was obtained from all the participants.

\section{Stimuli}

The stimuli were presented on a 20 -inch LaCie monitor (LaCie Electron 22 Blue IV) controlled by a Dell personal computer. We used the same visual stimuli and attractiveness pre-rating data as used in Park et al. (2010): Three categories of pictures - faces, natural scenes, and geometric figures - were used. The geometric figures were used as control, as Park et al. (2010) found them to elicit no strong choice bias. Faces were generated by FaceGen (Singular Inversions Inc.) software in 16 subcategories: 4 races (Asian, African, Indian, and European $) \times 2$ genders $\times 2$ ages (old and young). Natural scenes were color photos collected from public websites in eight subcategories: animal, food, flower, mountain, sky, lake, ocean, and desert. Geometric figures were Fourier descriptors generated by a Matlab program (MathWorks Inc.) with properties in one of the four combinations: symmetry vs. asymmetry $\times$ simple vs. complex. The geometric figures were randomly assigned into eight subcategories. Each subcategory of images contained 27 pictures and the one that received the median attractiveness rating was used as the "old," or repeated, picture. This was to avoid introducing an initial bias in preference choice toward the novel or familiar stimuli. 


\section{Design}

The experiment consisted of two phases: an experience phase (20 trials $\times 8$ subcategories $\times 3$ categories $)$ and a preference judgment phase ( 6 trials $\times 8$ subcategories $\times 3$ categories). In each phase, the three categories of pictures were run in different blocks, with the sequence of blocks randomly assigned to each participant. Within each block, the eight subcategories were run in different sub-blocks. Among the 16 available subcategories for faces, the eight assigned to a given participant were composed of faces of the participants' own race, and another randomly chosen race $(2$ races $\times 2$ genders $\times 2$ ages). For natural scenes and geometric figures, all eight subcategories in the image set were used. The order of subcategories was randomized within the block.

\section{Task}

During the experience phase, participants viewed pairs of pictures on the screen, with an unlimited, self-paced viewing time for each trial (however they were encouraged to just take glances). To encourage the participants to pay some attention to the stimuli, they were told that they would complete a questionnaire regarding the pictures afterward (but the questionnaire was not actually implemented). Participants were not informed of the existence of the preference judgment phase of the experiment until the experience phase was completed. In the preference judgment phase, participants were requested to make a relative preference judgment on each pair of pictures. The response was made by moving a cursor along an indicator frame to score the relative preference rating on a scale from 1 to 3 toward the right or left picture. Zero was allowed to indicate no preference for either picture (thus in effect, it was a 7-point scale).

Figure 1 shows the stimulus display and sample stimuli. In each trial, an old and a new picture were presented side-by-side on the screen. The left-right ordering of the old and new pictures was randomized in each trial. The center of each picture was located $10^{\circ}$ of visual angle from the central point. Face pictures were $12^{\circ} \times 12^{\circ}$, natural scenes were $15^{\circ} \times 12^{\circ}$ (horizontally oriented), and geometric figures were $17^{\circ} \times 12^{\circ}$. In the response phase, a response

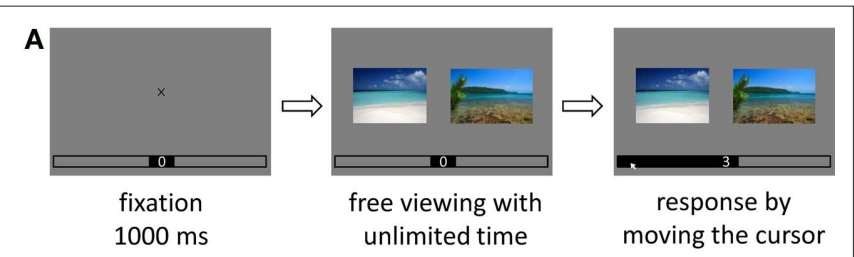

B

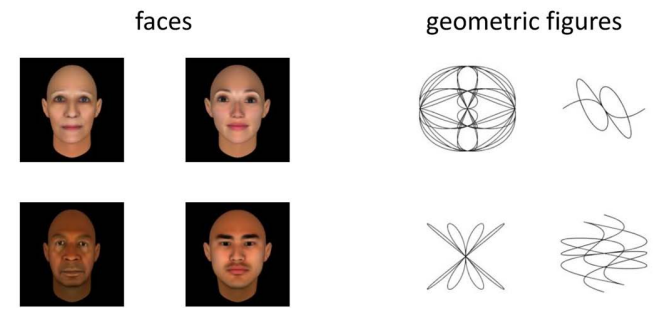

FIGURE 1 | Stimuli and procedure. (A) Sample judgment phase trial with natural scene images. (B) Examples of face and geometric figure images. indicator frame $\left(36^{\circ} \times 1.5^{\circ}\right)$ was located $12.7^{\circ}$ below to the central point. The indicator showed " 0 " initially in each trial and could be adjusted from " 1 " to " 3 " in either direction, right or left, to indicate the relative preference rating.

\section{RESULTS}

The preference rating was coded as a positive value (i.e., +1 to +3 ) if the repeated "old" picture was preferred, and negative value if the new picture was preferred. To evaluate the impact of the experience phase, we analyzed the first preference rating of each sub-block in the judgment phase (i.e., the first time a given old picture was subjected to a preference judgment). Figure 2 (black bars) shows the mean initial ratings within each stimulus category, with error bars indicating standard errors of the mean across participants. A one-way repeated-measures ANOVA against image category revealed that there were significant differences among the three object categories $[F(2,19)=6.94, \mathrm{MSE}=2.31, p<0.01]$ and Tukey's test showed a significant difference between faces and natural scenes $(p<0.01)$.

To evaluate whether there was any significant choice bias toward the familiar or novel picture, we tested the initial ratings for significant deviation from 0 with one-sample $t$-tests. The results showed that the familiar faces were significantly preferred $[t(19)=4.14$, $p<0.001$, Cohen's $d=0.93$ ], but no preference bias toward either familiarity or novelty was found in natural scenes $[t(19)=-1.49$, $p>0.1$, Cohen's $d=-0.33$ ] nor in geometric figures $[t(19)=1.51$, $p>0.1$, Cohen's $d=0.34]$.

As participants completed additional preference judgment trials on the paired stimuli, the preference for familiar faces increased further, and a preference for novel natural scenes emerged (Figure 3). By the sixth preference trial, novel natural scenes were significantly preferred over familiar ones $[t(19)=-2.57, p<0.02$, Cohen's $d=-0.58$, consistent with Park et al.'s (2010) original study.

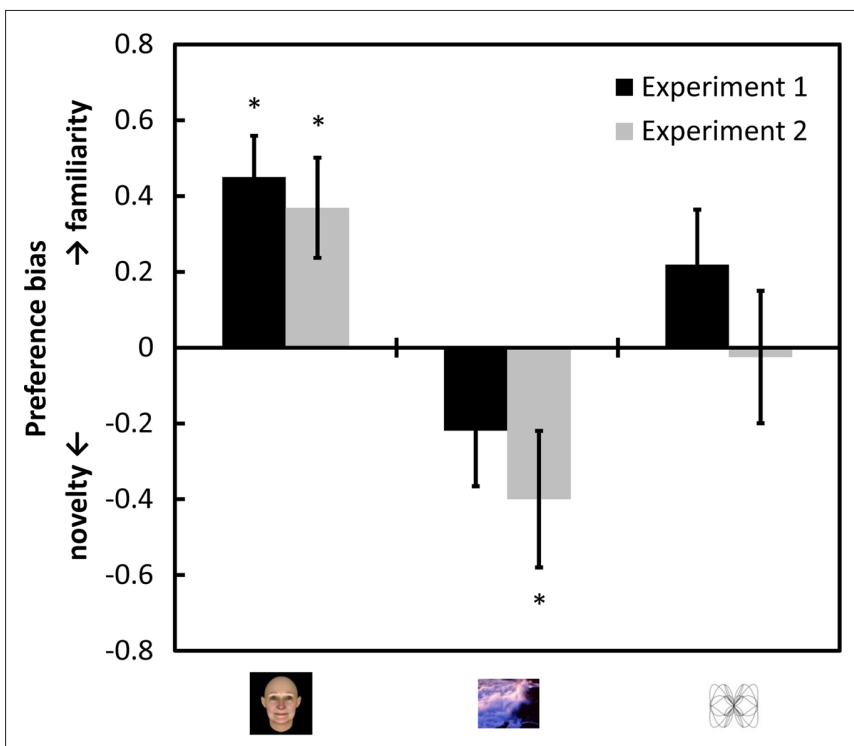

FIGURE 2 | Mean preference bias among initial preference judgments in Experiments 1 and 2. Error bars represent one standard error of the mean. Asterisks indicate statistically significant differences from 0. 


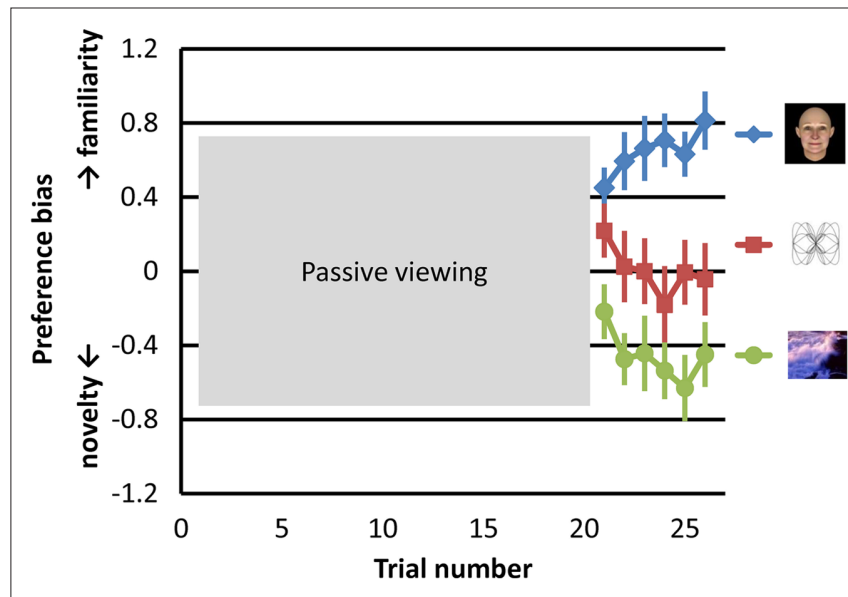

FIGURE 3 |Timelines of preference bias in Experiment 1. Data points are trial-by-trial means of ratings rectified toward the repeated image. Error bars represent one standard error of the mean.

\section{DISCUSSION}

The results showed that, while there was preference for familiar faces, no preference bias toward either familiarity or novelty was found in natural scenes immediately following the exposure phase, despite 20 repetitions of task-free exposure to each "old" picture. The result of natural scenes is inconsistent with the mere exposure effect (e.g., Zajonc et al., 1972), where repeatedly presented images were preferred; and also inconsistent with Park et al. (2010), where the repeatedly presented natural scenes were shown to be less preferred.

There are two alternative explanations as for why the mere exposure effect was not observed for natural scenes. One may result from the way the stimuli were presented in this study. Here, a pair of pictures was presented side-by-side, whereas in the typical mere exposure effect study (e.g., Zajonc, 1968; Zajonc et al., 1972; Bornstein, 1989), only one picture was viewed in each exposure. Paired presentations may lead to unbalanced examination durations if there is an attentional bias between the pictures. Another possibility is that the mere exposure effect may have been abolished by an overexposure effect (e.g., Zajonc et al., 1972; Williams, 1987) in the way that excessive exposure to the same stimulus induces aversion. These explanations do not account for the results of the face category, which showed familiarity preference after the same way and the same number of stimulus presentations as natural scenes, but it is possible that different object types may have different memory effects in preference (see more in General Discussion).

That there was no novelty preference bias in the natural scenes was inconsistent with Park et al. (2010), indicating that the development of a novelty preference for natural scenes requires the performance of the preference judgment task during exposure. Park et al. (2010) found a rapidly developing bias toward novelty preference that saturated after about five trials, and that sustained across at least 26 trials. With the preference task removed in our experiment, 20 trials of exposure still failed to elicit a significant preference bias. Further, our subsequent implementation of six preference judgment trials was sufficient to recover the preference bias effect to approximately the same magnitude as found in Park et al. (2010). This provides both negative and positive evidence that the novelty preference for natural scenes requires a task-context judgment during exposure.

\section{EXPERIMENT 2: TYPE OF JUDGMENT TASK}

Novelty preference for natural scenes was found when the subjective preference judgment is requested on each trial but not found after passive viewing experience, indicating that mere exposure is not sufficient to lead to novelty preference for natural scenes. It suggests that a deeper level of processing engaged by the selection task might be needed for novelty preference formation. However, it is unclear whether the necessary processing is specifically engaged by preference choices, or if the processing is engaged by selection tasks in general.

In this experiment, we examined this question by imposing objective judgment tasks (e.g., rating relative color of the natural scenes), rather than a subjective preference evaluation during the exposure phase. This evokes controlled selection processes without involving preference processes. If the judgment of preference needs to be carried out to elicit novelty preference for natural scenes, then objective judgment during the experience phase should not lead to novelty preference for natural scenes. However, if the conduct of any kind of selection judgment is sufficient to lead to novelty preference for natural scenes, a preference bias toward novel natural scenes is expected immediately after the experience phase.

We also examined how the objective evaluation would affect the preference for familiar faces, as the literature points in various directions. Halberstadt and Hooton (2008; also see Wilson et al., 1993) found that subjects prompted to report analytic reasons for liking or disliking a painting gave lower numeric ratings for how much they liked the painting, as compared to control subjects who only had to rate how much they liked the painting. In addition, the authors showed that there was a significant correlation between liking ratings and process fluency for control subjects, but this correlation disappeared in subjects who had been prompted for analytic reasoning. If analytic reasoning disrupts the connection between stimulus process fluency and the degree to which the stimulus is liked, then we would expect that the introduction of an objective task with analytic evaluation in our experiment might disrupt the preference for familiar faces. In contrast, according to the perceptual fluency account for familiarity preference (Mandler et al., 1987), it is possible that conducting the objective judgment may still facilitate perceptual fluency and thus lead to familiarity preference, just as repeated preference judgment does.

In this experiment, we examine the predictions from these hypotheses by introducing objective judgment during the experience phase. If the objective judgment disrupts stimulus process fluency, a disruption of the familiarity preference for faces is expected. Alternatively, if perceptual fluency is robust to task involvement, familiarity preference for faces should still be expected.

\section{METHODS}

Participants

Another group of 20 adults as described before participated in the main experiment. Five were Caucasians and the rest were Asians. Another group of 10 adults pre-rated the stimuli for objective attributes. 


\section{Stimuli, design, and procedure}

Stimuli, design, and procedure were the same as in Experiment 1, except for the following: First, the pictures chosen to be "old" or repeated pictures in this experiment were the ones receiving median ratings both in terms of their attractiveness and their objective attributes. This was done so that the stimulus itself would not bias choice toward the old or new picture in either the objective or preference judgment phases. In pre-rating sessions, participants were asked to rate with a 7-point scale (from 1 to 7 ): the roundness of each face, the overall color-temperature of each natural scene (higher score $=$ warmer color content, e.g., redness), and the complexity of each geometric figure. All the pictures were ranked for both attractiveness and objective attributes within each subcategory, and pictures possessing median ratings in both rankings were selected as the "old" pictures.

Second, in the main experiment, during the experience phase, participants were required to make a relative judgment regarding an object feature for each pair of pictures. For faces they had to rate their relative roundness (from +3 to -3 ), for natural scenes their relative overall color-temperature, and for geometric figures their relative complexity.

\section{RESULTS}

Results are shown in Figure 4. As expected, during the experience phase where objective judgments were performed, there was no systematic tendency for ratings toward familiar or novel pictures by exposed trials in any object categories (all $R^{2} s<0.2, p s>0.07$ ). In contrast, there was a progressively accumulating preference bias toward familiar faces and novel natural scenes during preference judgment phase (both $R^{2} s>0.7$, ps $<0.03$ ).

Figure 2 (gray bars) shows the mean coded ratings given for the initial preference trials. An ANOVA revealed the main effect of object categories $[F(2,19)=5.62, \mathrm{MSE}=2.96, p<0.01]$ and Tukey's test revealed a significant difference between faces and natural scenes $(p<0.01)$. One-sample $t$-tests of the initial ratings showed that, preference bias toward familiar faces $[t(19)=2.79, p<0.02$, Cohen's $d=0.62]$ and toward novel natural scenes $[t(19)=-2.22$,

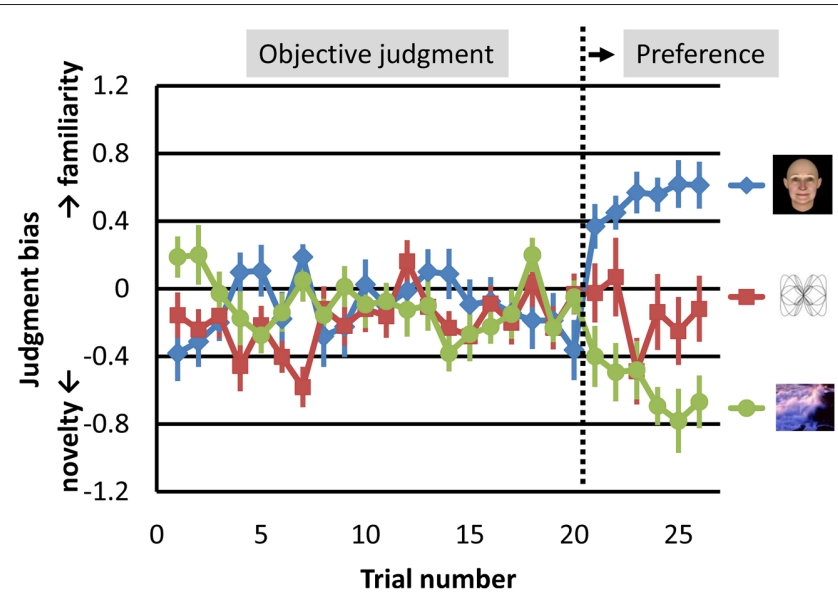

FIGURE 4 |Timelines of rating bias in Experiment 2. Data points are trial-by-trial means of ratings rectified toward the repeated image. Error bars represent one standard error of the mean. $p<0.04$, Cohen's $d=-0.50$ ] was immediately present after the experience phase with objective judgments, but no bias was present for geometric figures $[t(19)=-0.14, p>0.8$, Cohen's $d=-0.03$ ].

To further examine whether depth of processing or selection made a difference in preference bias for familiar face and novel natural scenes, we conducted a direct comparison between the two experiments. An ANOVA on the initial preference ratings with the three object categories as the within-subject variable and the two experiments as the between-subject variable showed the main effect of object category $[F(2,76)=12.10, \mathrm{MSE}=5.20, p<0.001]$, but not the main effect of experiment $[F(1,38)=1.74, \mathrm{MSE}=0.85, p>0.1]$ nor the two-way interaction $[F(2,76)=0.16, \mathrm{MSE}=0.07, p>0.8]$.

\section{DISCUSSION}

The results showed that after the experience phase, which involved the objective evaluation of the color-temperature of the pictures, novel natural scenes were immediately preferred over the familiar ones. Whereas Experiment 1 indicated that a judgment task was necessary for stimulus experience to elicit novelty preference for natural scenes, this experiment indicates that the judgment task need not be one based on preference. This experiment also replicates the finding of familiarity preference for faces, as well as the significant difference between face and natural scene stimuli.

Regarding the familiarity preference for faces, we did not find evidence that the objective evaluation of visual features (roundness) disrupted process fluency or its connection to the preference response. However, other studies have shown that analyzing and reasoning the liking (e.g., Wilson et al., 1993; Halberstadt and Hooton, 2008) has such an effect. The discrepancy between the current result and previous studies in preference formation may be explained by that objective judgment on just a single visual feature is not sufficient to interfere with preference attitudes. Alternatively, it may be differences between the tasks in processing depth, time, or effort. Yet another possibility would be that there is something special in faces (see review in Kanwisher and Yovel, 2006) that leads to a more robust familiarity preference. In any case, the current result is consistent with the perceptual fluency account (Mandler et al., 1987) in that the familiarity preference for faces is caused by the general process fluency at the perceptual level and is not affected by specific type of objective evaluation.

In post hoc interviews of participants in the two experiments, all of the participants noticed that there was a repeatedly presented stimulus but none of them reported that their preference choice was based on it. This suggests that the preference biases found here may be driven by implicit processing (e.g., Shimojo et al., 2003; Johansson et al., 2005). When asked for the reasons behind their preferences, some participants named random attributes such as the brightness of the picture, a cheerful feeling, or just intuition. Whether familiarity preference for faces and novelty preference for natural scenes are modulated by different mechanisms or different depths of processing (or both), it appears that experience affects the preference through such implicit processing.

\section{GENERAL DISCUSSION}

In two experiments, repeated exposure to a face led to a preference bias toward that face whether or not a judgment task was conducted during stimulus exposure. In contrast, repeated exposure to 
a natural scene led to a preference bias away from that scene only when a selection task (whether an objective judgment or subjective preference judgment) was conducted during exposure. The results suggest that passive perceptual exposure without a task is sufficient to induce the familiarity preference for old faces, but a certain level of processing or selection seems to be required to induce the novelty preference for new natural scenes. Thus the task-context of experience has different influences on preferences, and needs to be accounted for, to further understand the mechanisms underlying familiarity and novelty preferences.

One possibility is that experience drives familiarity preference for faces and novelty preference for natural scenes via different mechanisms. It is raised by the original finding of the preference segregation, i.e., the familiarity vs. the novelty preferences across object categories, in that the same experience can have opposite directions of influences on preference depending on the object type. Furthermore, the results in Experiment 1 that after passive exposure only familiar faces but not novel natural scenes were preferred indicate that the two types of preferences are influenced by the passively exposed experience differently.

Alternatively, the two types of preferences may involve the same or similar mechanism during exposure but are modulated by the depth of processing or involvement of selection processing. One way to examine this issue is to make a direct comparison between Experiment 1 (passive exposure without judgment tasks) and Experiment 2 (objective judgment tasks) to test whether the task-context manipulation between the two experiments results in different effects. If different mechanisms are involved, different experience effects may be expected between the two experiments. The direct comparison actually did not reveal a statistical difference. However, the results may have been obscured by several factors. Just to name a few, the comparison was made between different groups of participants, it was based on only a few trials contributed by each participants, and the statistical power may be thus reduced.

Another important factor to obscure the results may be the different objective judgment tasks across object categories in Experiment 2: roundness for faces and color-temperature for natural scenes. The stimulus attributes between faces and natural scenes are inherently different and this may constrain the objective judgment involvement to confound with object types. In order to make the objective task more natural and functionally relevant to each category, different types of objective judgment are implemented with different object types. This inevitable choice of the task, however, made it difficult to directly compare the results and draw a strong conclusion.

Many explanations have been proposed to account for the tendency to prefer familiar items. For example, the perceptual fluency account is that the ease of processing familiar items reduces cognitive load and thus causes relaxation and other responses related to positive affect (Jacoby and Dallas, 1981; Bornstein and D'Agostino, 1994). Alternatively, Zajonc (2001) proposed a conditioning-based explanation, based on the absence of noxious events across the repeated stimuli. In this case, a positive affect, or safety signal, due to the absence of aversive consequences is associated with the repeated stimulus, establishing a linkage between the repeated stimulus and preference. There are also studies showing that familiarity preference is modulated by other factors such as mood (de Vries et al., 2010) or social context (Crisp et al., 2009).

We build on these perspectives by examining how preferences are affected by task-context, which gives us clues as to the types of memory processes involved. Our results demonstrate that familiarity preference for faces is formed regardless whether the processing involves mere exposure, objective judgment, or subjective preference judgment. This suggests that familiarity preference for faces may result from more bottom-up, lower-level, and non-selective perceptual facilitation. This is consistent with the evidence showing that familiarity preference is less correlated with explicit memory and more related to implicit memory (Bornstein and D'Agostino, 1992). The dissociation of familiarity preference and explicit memory recognition has also been shown in taste (Adolphs et al., 2005) and music (Szpunar et al., 2004).

Novelty preference for natural scenes, on the other hand, seems to require a certain level of processing depth or selection. This may be related to the biased competition hypothesis for novelty preference (Snyder et al., 2008), which suggests that novel stimuli elicit stimulus-driven attentional biases (i.e., novelty attracts attention) and that this induces the preference. There is also evidence for novelty place preference in animals, which appears to be based on novelty seeking rather than familiarity avoidance (Klebaur and Bardo, 1999). In accordance with the biased competition hypothesis, Raymond et al. (2003) showed that, when repeated stimuli are ignored, they end up less preferred than attended or novel stimuli, indicating that attention modulates the way experience affects preferences. These studies show that novelty preference is related to attentional selection bias, which is consistent with our results. It is possible that the repeated natural scenes are less preferred because repetition leads to a habituation of attention toward the old stimulus, and attention is thus biased toward the novel natural scenes. In conditions where there is no selection task during exposure, attention is less engaged, reducing the biasing effects on preference.

Preference for familiarity vs. novelty may be understood as an inverted- $U$ function between preference and frequency of stimulus exposure. For example, Zajonc et al. (1972) presented colorful paintings and varied the frequency of stimulus exposure between $1,2,5,10$, and 25 times across subjects. After the exposure, subjects were asked to rate how much they liked each painting. Results showed that preference ratings increased with small numbers of exposures, but began to decline with larger numbers of exposures, eventually falling below the level of novel stimuli by the $25^{\text {th }}$ exposure. If translated into our preference choice setting, the results indicate familiarity preference with lower numbers of exposures and novelty preference with higher numbers of exposures.

An intriguing aspect of that study is that the peak of the inverted$\mathrm{U}$ function was found to shift with the degree of similarity among the paintings that were exposed and rated. The paintings in the first experiment of Zajonc et al. (1972) were distinctively different from each other, and ratings peaked after one to two exposures. However, when multiple portions of the same painting were used to create a stimulus set with low visual variability, ratings peaked at 10 exposures or more, and the ratings never declined below the level of novel stimuli, even at 25 exposures. Likewise when they used photographs of faces, they found only familiarity preference, 
which continued to increase even up to 25 exposures. Altogether, the peak of the familiarity bias appears to shift later as the similarity between stimuli in the set increases. Thus, the pattern of familiarity and novelty preferences emerging from stimulus experience may be affected by interactions between type and similarity, which is consistent with the segregation in preferences across object categories, reported by Park et al. (2010) and duplicated in the current study. Faces are relatively similar to each other in terms of low-level visual features, as compared to those of natural scenes (see Park et al. (2010) for more detailed discussion), which might lead to early reversals toward novelty preference for natural images, and longer-term maintenance of familiarity preference for faces.

The emerging picture from the related studies and the current results is that difference between familiarity preference for faces and novelty preference for natural scenes may result from the interaction between the task-context experience and object categories. For example, it could be possible that faces are biologically special and mutually similar, to form familiarity preference, as suggested by the conditioning with safety signal (e.g., Zajonc, 2001; de Vries et al., 2010), and therefore the familiarity preference for faces is resistant to task-context experience manipulation. In contrast, natural scenes are with large varieties and more susceptible to processing depth or selection to affect preference formation.

\section{REFERENCES}

Adolphs, R., Tranel, D., Koenigs, M., and Damasio, A. R. (2005). Preferring one taste over another without recognizing either. Nat. Neurosci. 8, 860-861.

Berlyne, D. E. (1970). Novelty, complexity, and hedonic value. Percept. Psychophys. 8, 279-286.

Bevins, R. A., and Bardo, M. T. (1999). Conditioned increase in place preference by access to novel objects: antagonism by MK-801. Behav. Brain Res. 99, 53-60.

Bornstein, R. F. (1989). Exposure and affect: overview and meta-analysis of research, 1968-1987. Psychol. Bull. 106, 265-289.

Bornstein, R. F., and D'Agostino, P. R. (1992). Stimulus recognition and the mere exposure effect. J. Pers. Soc. Psychol. 63, 545-552.

Bornstein, R. F., and D'Agostino, P. R. (1994). The attribution and discounting of perceptual fluency: preliminary tests of a perceptual fluency/attributional model of the mere exposure effect. Soc. Cogn. 12, 103-128.

Crisp, R. J., Hutter, R. R. C., and Young, B. (2009). When mere exposure leads to less liking: the incremental threat effect in intergroup contexts. Br. J. Psychol. 100, 133-149.

de Vries, M., Holland, R. W., Chenier, T., Starr, M. J., and Winkielman, P. (2010). Happiness cools the warm glow of familiarity: psychophysiological evidence that mood modulates the familiarity-affect link. Psychol. Sci. 21, 321-328.
Fantz, R. L. (1964). Visual experience in infants: decreased attention to familiar patterns relative to novel ones. Science 146, 668-670.

Garcia-Marques, T., Mackie, D. M., Claypool,H.M., and Garcia-Marques, L. (2010). Is it familiar or positive? Mutual facilitation of response latencies. Soc. Cogn. 28, 205-218.

Halberstadt, J., and Hooton, K. (2008). The affect disruption hypothesis: the effect of analytic thought on the fluency and appeal of art. Cogn. Emot. 22, 964-976.

Houston-Price, C., and Nakai, S. (2004) Distinguishing novelty and familiarity effects in infant preference procedures. Infant Child Dev. 13, 341-348.

Hupbach, A., Melzer, A., and Hardt, O. sensitive to color information: evidence for color effects in a perceptual implicit memory test. Exp. Psychol.53, 233-245.

Jacoby, L. L., and Dallas, M. (1981). On the relationship between autobiographical memory and perceptual learning. J. Exp. Psychol. Gen. 110, 306-340.

Johansson, P., Hall, L., Sikstrom, S., and Olsson, A. (2005). Failure to detect mismatches between intention and outcome in a simple decision task. Science 310, 116-119.

Kanwisher, N., and Yovel, G. (2006). The fusiform face area: a cortical region specialized for the perception of faces. Philos. Trans. R. Soc. Lond., B, Biol. Sci. $361,2109-2128$ (2006). The mere exposure effect is

In summary, the current study showed how the task-context of stimulus exposure affects preference: Familiar faces were preferred whenever the faces had previously been presented, whether those exposures occurred in the context of passive viewing or explicit comparisons. On the other hand, novel natural scenes became preferred only when the exposures occurred in the context of explicit comparisons. The comparison conducted during exposure did not have to be preference based - objective comparisons of a visual feature also elicit novelty preference. The overall results indicate that the formation of a preference for familiarity or novelty depends on interactions of several factors, such as task-context, processing level, stimulus type, and similarity.

\section{ACKNOWLEDGMENTS}

We thank Junghyun Park for providing the stimuli from his original study, and Daw-An Wu for editing assistance. This research was supported by Japan Science and Technology Agency Exploratory Research for Advanced Technology (Shimojo Implicit Brain Function Project), National Institutes of Health Grant EY013274, and National Science Council in Taiwan (NSC 96-2413-H-002009-MY3, 98-2410-H-002-023-MY3, 098-2811-H-002-034, and 099-2811-H-002-038).

Klebaur, J.E., and Bardo, M. T. (1999). The effects of anxiolytic drugs on noveltyinduced place preference. Behav. Brain Res. 101, 51-57.

Mandler, G., Nakamura, Y., and Vanzandt, B. J. S. (1987). Nonspecific effects of exposure on stimuli that cannot be recognized. J. Exp. Psychol. Learn. Mem. Cogn. 13, 646-648.

Monahan, J. L., Murphy, S. T., and Zajonc, R. B. (2000). Subliminal mere exposure: specific, general, and diffuse effects. Psychol. Sci. 11, 462-466.

Olst, E. H. (1971). The Orienting Reflex. New York: Walter De Gruyter Inc.

Park, J., Shimojo, E., and Shimojo, S (2010). Roles of familiarity and novelty in visual preference judgments are segregated across object categories. Proc. Natl. Acad. Sci. U.S.A. 107, 14552-14555.

Raymond, J. E., Fenske, M. J., and Tavassoli, N. T. (2003). Selective attention determines emotional responses to novel visual stimuli. Psychol. Sci. 14 537-542.

Schneider, W., and Shiffrin, R. (1977) Controlled and automatic human information processing: I. Detection, search, and attention. Psychol. Rev. 84, 1-66.

Shiffrin, R., and Schneider, W. (1977) Controlled and automatic human information processing: II. Perceptual learning, automatic attending, and a general theory. Psychol. Rev. 84 127-190.

Shimojo, S., Simion, C., Shimojo, E., and Scheier, C. (2003). Gaze bias both reflects and influences preference. Nat. Neurosci. 6, 1317-1322.

Snyder, K. A., Blank, M. P., and Marsolek, C. J. (2008). What form of memory underlies novelty preferences? Psychon. Bull. Rev. 15, 315-321.

Szpunar, K. K., Schellenberg, E. G., and Pliner, P. (2004). Liking and memory for musical stimuli as a function of exposure. J. Exp. Psychol. Learn. Mem. Cogn. 30, 370-381.

Topolinski, S., and Strack, F. (2009). Motormouth: mere exposure depends on stimulus-specific motor simulations. J. Exp. Psychol. Learn. Mem. Cogn. 35, 423-433.

Williams, S. M. (1987). Repeated exposure and the attractiveness of synthetic speech: an inverted-U relationship. Curr. Psychol. Res. Rev. 6, 148-154.

Wilson, T. D., Lisle, D. J., Schooler, J. W., Hodges, S. D., Klaaren, K. J., and Lafleur, S. J. (1993). Introspecting about reasons can reduce post-choice satisfaction. Pers. Soc. Psychol. Bull.19, 331-339.

Wu, S. C., and Remington, R. W. (2003). Characteristics of covert and overt visual orienting: evidence from attentional and oculomotor capture. J. Exp. Psychol. Hum. Percept. Perform. 29, 1050-1067.

Zajonc, R. B. (1968). Attitudinal effects of mere exposure. J. Pers. Soc. Psychol. Monogr. Suppl. 9, 1-27.

Zajonc, R. B. (2001). Mere exposure: gateway to the subliminal. Curr. Dir. Psychol. Sci. 10, 224-228. 
Zajonc, R. B., Shaver, P., Tavris, C., and van Kreveld, D. (1972). Exposure, satiation, and stimulus discriminability. J. Pers. Soc. Psychol. Monogr. Suppl. 21, 270-280.

Conflict of Interest Statement: The authors declare that the research was conducted in the absence of any commercial or financial relationships that could be construed as a potential conflict of interest.

Received: 10 September 2010; accepted: 02 March 2011; published online: 18 March 2011.
Citation: Liao H-I, Yeh S-L and Shimojo $S$ (2011) Novelty vs. familiarity principles in preference decisions: task-context of past experience matters. Front. Psychology 2:43. doi: 10.3389/fpsyg.2011.00043

This article was submitted to Frontiers in Cognitive Science, a specialty of Frontiers in Psychology.
Copyright $\odot 2011$ Liao, Yeh and Shimojo. This is an open-access article subject to an exclusive license agreement between the authors and Frontiers Media SA, which permits unrestricted use, distribution, and reproduction in any medium, provided the original authors and source are credited. 